\title{
Die alte Wallonen-Eiche von Tricase oder „Die Eiche der hundert Ritter“
}

\author{
Katharina Kamm
}

\begin{abstract}
„La quercia dei cento Cavalieri“ is a several hundred years old Valonic oak (Quercus ithaburensis ssp. macrolepis). It grows in Apulia. There are many legends about this impressive tree. The Valonic oak is distributed from Italy to the Balkans and Turkey. The cupula is quite large and scaly. This oak species is hardy and is also found in the Palmengarten.
\end{abstract}

\section{Zusammenfassung}

„La quercia dei cento Cavalieri“ ist eine mehrere hundert Jahre alte Wallonen-Eiche (Quercus ithaburensis ssp. macrolepis). Sie wächst in Apulien, viele Sagen ranken sich um diesen eindrucksvollen alten Baum. Die Wallonen-Eiche kommt über den Balkan bis in die Türkei vor. Auffällig ist ihre große, schuppige Cupula. Diese Eichen-Art gedeiht auch im Palmengarten.

\section{1. „La quercia dei cento Cavalieri“", ein Eichen-Veteran in Apulien}

Kaum zu übersehen, aber doch irgendwie versteckt zwischen den zwei Spuren einer Landstraße, etwa anderthalb Kilometer von der Küste entfernt in der apulischen Gemeinde Tricase in Italien steht eine beachtliche alte Wallonen-Eiche (Quercus ithaburensis ssp. macrolepis). Sie trägt den italienischen Spitznamen „La quercia dei cento Cavalieri“, zu deutsch „Die Eiche der hundert Ritter/Reiter“. Der Baum ist geschätzt 700 Jahre alt mit einer $700 \mathrm{~m}^{2}$ großen Krone und einem Stammdurchmesser von 4,25 m (gianttrees.org).

Zur Herkunft des Spitznamens gibt es mehr als eine Legende. Eine dreht sich um Friedrich II., der unter der Eiche wahlweise seine Armee versammelte (treeoftheyear.org), mit hundert Rittern

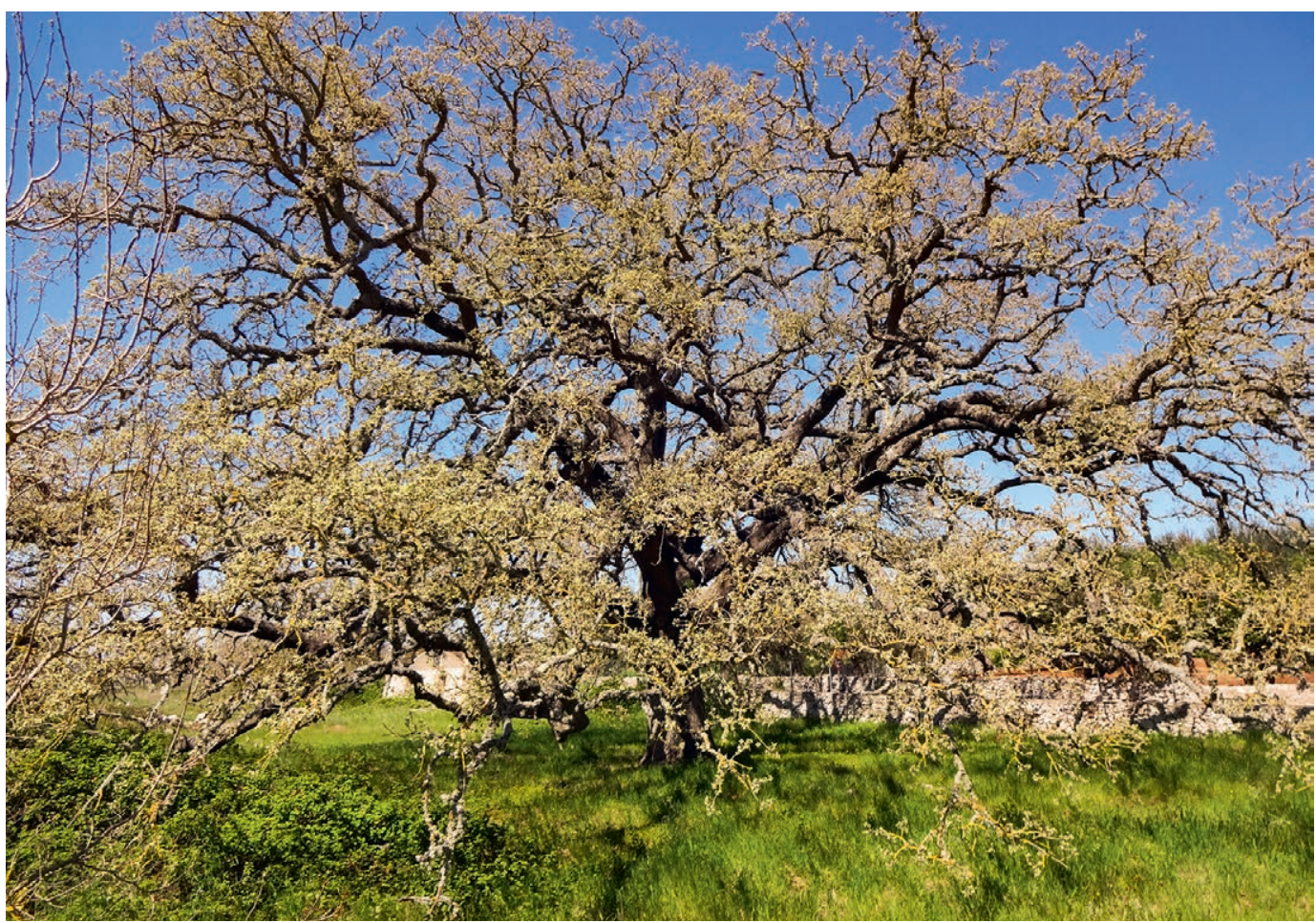

Abb. 1: „La quercia dei cento Cavalieri“ Ende März. (Foto: K. Kaмm) 


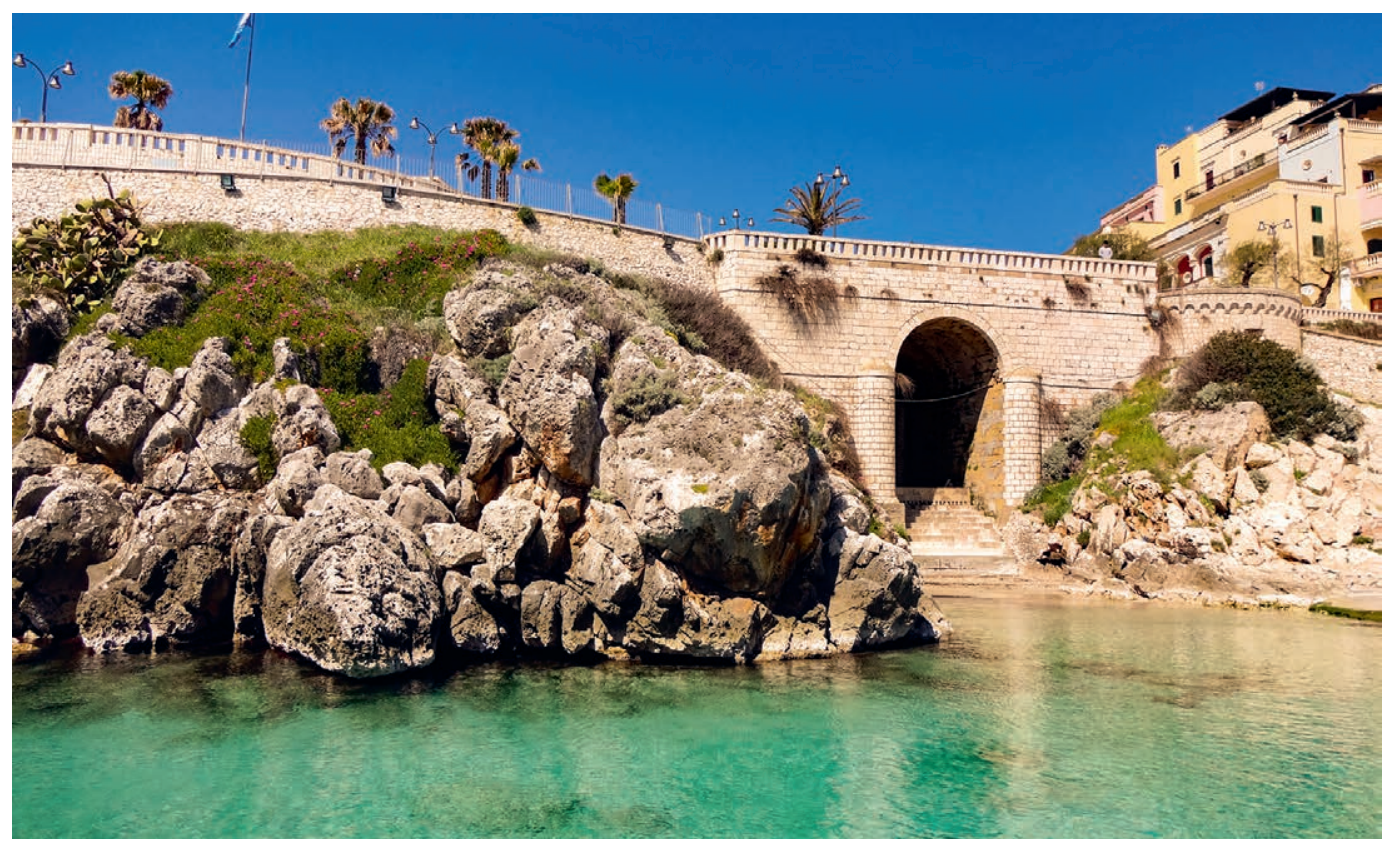

Abb. 2: Castro Marina an der Ostküste Apuliens, etwa 9 km nördlich der „quercia dei cento Cavalieri“. (Foto: K. Kamm)

Schutz vor einem Sturm suchte (gianttrees.org) oder von den Kreuzzügen zurückkehrend mit hundert Rittern und seinem gesamten Hofstaat Platz unter dem Kronendach fand (IAIA 2018). Da FRIEDRICH II. allerdings bereits 1250 verstarb, müsste die Eiche wohl weit über 800 Jahre alt sein, um ihm und seinen Gefolgsleuten bereits damals unter ihrer Krone genug Platz bieten zu können.

Einer anderen Legende zufolge wäre sie sogar noch älter. Denn diese besagt, dass vor fast 1000 Jahren dem Prinzen von Tricase nach jahrelangen Auseinandersetzungen nur eine Armee von 100 Mann blieb. Doch auch als sich die nächste Schlacht ankündigte, waren seine tapferen Soldaten bereit, wieder für ihn in den Krieg zu ziehen. Da dem Prinzen bewusst war, dass der Kampf aussichtslos war, schenkte er jedem seiner Ritter eine Eichel. Diese sollten die Männer schützen, bis sie wieder zu Hause ankamen. Die Ritter pflanzten jedoch die hundert Eicheln ein und baten Mutter Natur, aus ihnen gemeinsam eine einzige Eiche wachsen zu lassen. An diesem Ort wollten sich die Seelen der Ritter wieder treffen, falls sie nicht aus der Schlacht zurückkehren würden. Die Soldaten kehrten tatsächlich nicht zurück, aber die Natur kam ihrer Bitte nach (IAIA 2018).

\section{Wallonen-Eichen}

Die Wallonen-Eiche hat ein Verbreitungsgebiet von Albanien und Griechenland über die Türkei (auch wenn sie dort seltener sind) bis nach Israel und Jordanien. Sie wächst dort vorwiegend in lichten Wäldern oder Dickichten (Bussotтı \& Bоттассі 2003). Die Bäume im süditalienischen Apulien stammen vermutlich von griechischen Siedlern oder aus albanischem Saatgut (AvishaI 2016, Bussotтi \& Воттассі 2003).

Quercus ithaburensis ssp. macrolepis wird etwa 10 bis $15 \mathrm{~m}$ (im Extremfall $26 \mathrm{~m}$ ) hoch mit breiten Kronen. Die Stämme erreichen Durchmesser bis $60 \mathrm{~cm}$ und entwickeln eine dunkelbraune, tief längsrissige Borke. Die Bäume zeigen ein flaches, aber weites Wurzelsystem (Bussotтi \& Bоттассі 2003).

Ihre Blätter sind beim Austrieb weich und behaart, verkahlen später auf der Oberseite größtenteils und werden ledrig derb. Sie werden 6 bis 7 (10) $\mathrm{cm}$ lang und 3 bis 4 (8) $\mathrm{cm}$ breit mit einem 


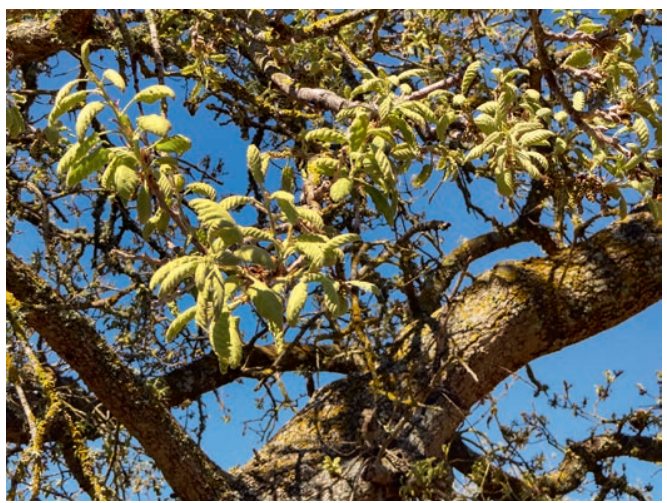

Abb. 3: Austrieb der Wallonen-Eiche. (Foto: K. Камм)

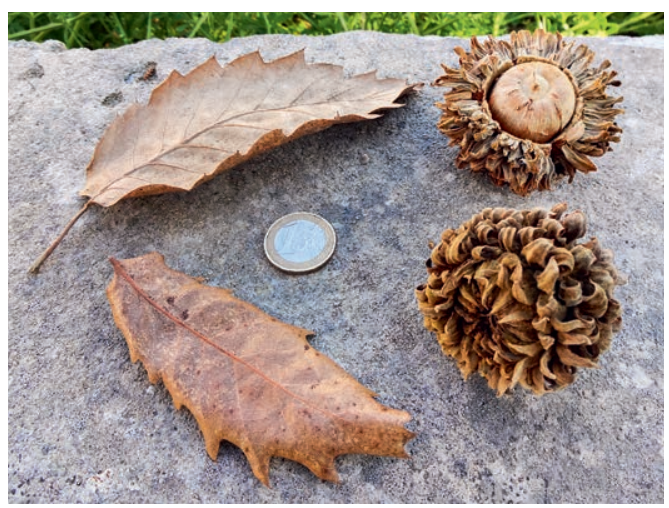

Abb. 4: Laub und Eicheln der Wallonen-Eiche.

(Foto: K. Камм)

bis $3 \mathrm{~cm}$ langen Stiel (Bussotti \& Bottacci 2003). Sie sind unregelmäßig gelappt, die bis 10 Lappen pro Seite enden in spitzen Zähnen (Coombes 2010). Die Blätter werden erst zum Ende des Winters abgestoßen (Bussotтi \& Воттассі 2003).

Die Eiche blüht im April oder Mai mit männlichen und weiblichen Blütenständen am selben Baum. Die männlichen Blütenstände sind kätzchenförmig, die weiblichen kurz ährenförmig. Die gestielten Eicheln, die erst im Herbst des folgenden Jahres reif werden, stehen allein oder in Gruppen bis zu 5 (Bussotti \& Bоттассі 2003). Die Eicheln sind zum Großteil von einer schuppigen Cupula (d. h. einem Fruchtbecher) umgeben, der über $5 \mathrm{~cm}$ im Durchmesser messen kann. Gemeinsam sind Eicheln und Cupulae etwa $4,5 \mathrm{~cm}$ lang, wobei nur etwas mehr als $1 \mathrm{~cm}$ der Eichel nicht von der Cupula eingeschlossen wird

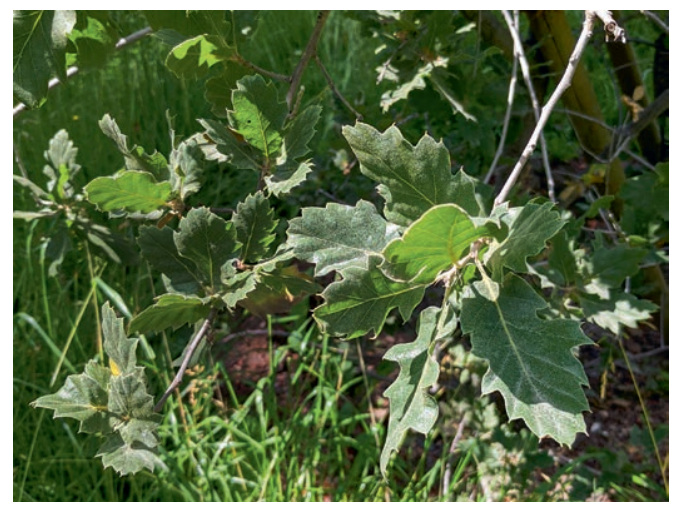

Abb. 5: Blätter einer Wallonen-Eiche im Palmengarten im Juni. (Foto: K. Камм)

(Dufort-Dror \& Ertas 2002). Die basalen Schuppen der Cupula stehen aufrecht, die mittleren haben oft einen einwärts gebogenen Rand und die Schuppen am Rand des Fruchtbechers liegen der Eichel an (Bussotti \& Bоттассі 2003).

Die Cupulae haben einen hohen Gerbstoffanteil von bis $30 \%$. Sie wurden unter der Bezeichnung Orientalische Knoppern zum Gerben und Färben von Leder eingesetzt (Sронn \& Spoнn 2011). Ende des 19. Jahrhunderts wurden sogar Eicheln und Jungpflanzen nach Australien verschifft, um dort Plantagen einzurichten (CAMERon 2014). Eine der drei noch erhaltenen Standorte wurde mit Hilfe von Schulkindern mit jungen WallonenEichen wieder aufgeforstet (CAMEron 2016). Mit den ebenso als Knoppern bezeichneten Gallen, die manchmal an den Eicheln von Stiel- oder TraubenEichen zu finden sind, haben die Orientalischen Knoppern übrigens nichts zu tun. Heute erfreuen sich auf diese Weise gegerbte Schuhe neuer Beliebtheit (Sронn \& Sронn 2011).

Die systematische Einordnung der WallonenEiche ist seit ihrer Erstbeschreibung als Quercus macrolepis durch TheOdoR Kотschy im Jahr 1860 vielen Veränderungen unterworfen (AvisHAI 2016). Derzeit wird das Taxon oft als Unterart von Quercus ithaburensis behandelt. Genetischen Untersuchungen zufolge sollte die Wallonen-Eiche jedoch wieder als eigenständige Art (Quercus macrolepis) angesehen werden (SimEONE et al. 2018). Sie gehört zur Sektion der Zerr-Eichen. 


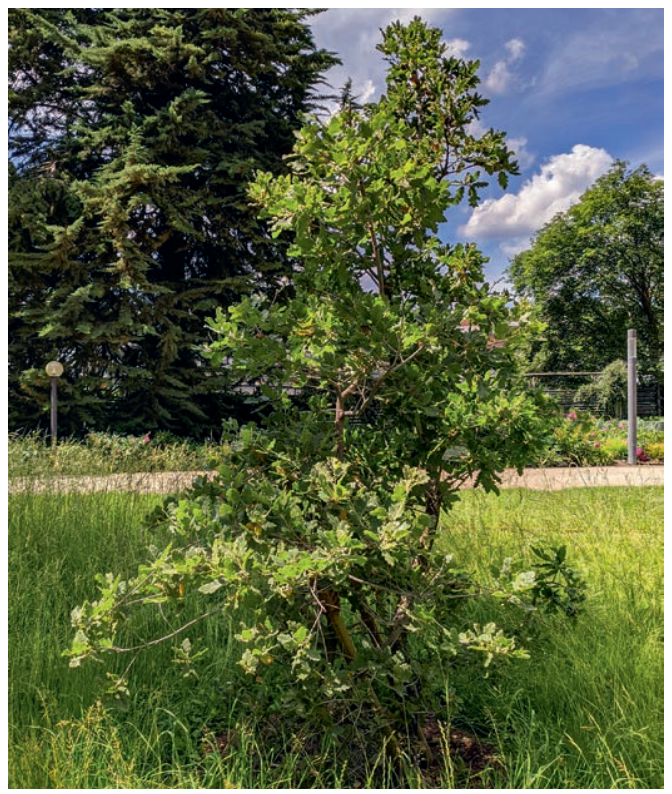

Abb 6: Es wird wohl noch etwas dauern, bis unter dieser jungen Wallonen-Eichen im Palmengarten 100 Leute Platz finden. (Foto: K. Камм)

Man muss nicht bis nach Italien reisen, um Wallonen-Eichen zu sehen. Ein schönes, noch junges Exemplar wächst in der Nähe des Staudengartens. Eine zweite Eiche ist zwar deutlich älter, wächst unscheinbar in einer Hecke in der Nähe des Sommer-Sukkulentengartens und bleibt strauchförmig.

\section{Literatur}

Bussotti, F \& Bоттассі, A. 2003: Quercus macrolepis. In: Roloff, A., Weisgerber, H., Lang, U. M. \& Stimm, B. (Hrsg.): Enzyklopädie der Holzgewächse. Handbuch und Atlas der Dendrologie. - Weinheim.

Coombes, A. 2010: The book of leaves. A leaf-by-leaf guide to six hundred of the world's great trees. - Chicago.

Dufour-Dror, J-M. \& Ertas, A. 2002: Cupule and acorn basic morphological differences between Quercus ithaburensis Decne. subsp. ithaburensis and Quercus ithaburensis subsp. macrolepis (Kotschy) Hedge \& Yalt. - Acta Botanica Malacitana, 27: 237-242.

Simeone, M. C., Cardoni, S., Piredda, R., Imperatori, F, Avishai, M. Grimm, G. W. \& Denk, T. 2018: Comparative systematics and phylogeography of Quercus Section Cerris in western Eurasia: inferences from plastid and nuclear DNA variation. PeerJ 6: e5793 https://doi.org/10.7717/peerj.5793

Spohn, M. \& Spohn, R. 2011: Kosmos-Baumführer Europa. - Stuttgart.

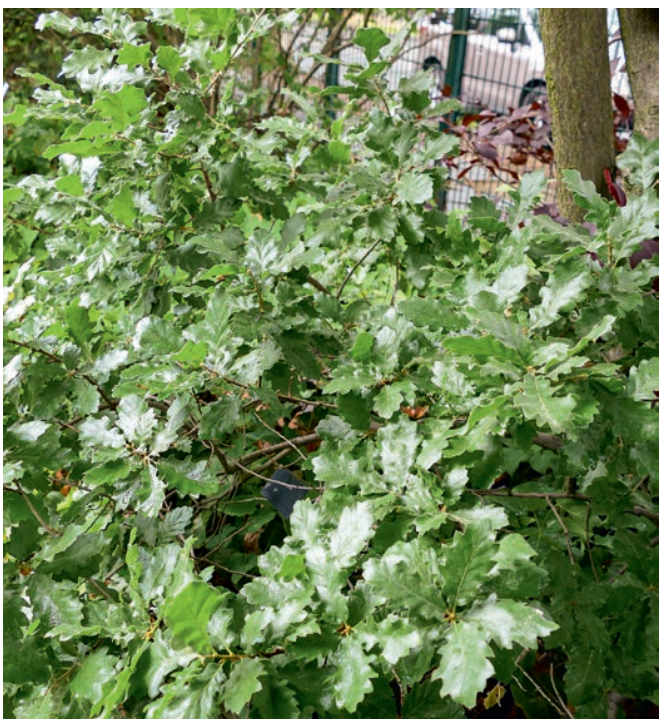

Abb. 7: Diese kleine Wallonen-Eiche wächst schon viele Jahre lang in einer Hecke an der Gartengrenze zur Siesmayerstraße. Sie wird zwar kaum größer, hat aber die kalten Winter Anfang des 21. Jahrhunderts gut überstanden. (Foto: H. Steinecke)

\section{Internetseiten:}

Avishai, M. 2016: Vallonea or Aegilops Oaks, a Short Review. https://www.internationaloaksociety.org/content/vallonea-oraegilops-oaks-short-review

Cameron, R. 2014: Valonia Oak Restoration Project, Castlemaine, Victoria. https://www.internationaloaksociety.org/ content/valonia-oak-restoration-project-castlemaine-victoria

Cameron, R. 2016: Update on the Valonia Oak Restoration Project. https://www.internationaloaksociety.org/content/update-valonia-oak-restoration-project

IAIA, A. 2018: La leggenda della quercia dei cento cavalieri. https:// www.ilsudchenontiaspetti.it/quercia-dei-cento-cavalieri/ https://www.gianttrees.org/en/tree-of-the-year/tree-of-the-year-2019

https://www.treeoftheyear.org/Stromy/Dub-Vallonea-z-Tricasu http://powo.science.kew.org/taxon/296425-1

\section{Anschrift der Autorin}

Katharina Kamm, Palmengarten Frankfurt, Siesmayerstr. 61, 60323 Frankfurt, E-Mail: katharina.kamm@stadt-frankfurt.de 